\title{
Meta-analysis of associations between TCF7L2 polymorphisms and risk of type 2 diabetes mellitus in the Chinese population
}

Jinjin Wang ${ }^{1}$, Fulan $\mathrm{Hu}^{2}$, Tianping Feng ${ }^{3+}$, Jingzhi Zhao ${ }^{3+}$, Lei Yin ${ }^{3+}$, Linlin $\mathrm{Li}^{1+}$, Yan Wang ${ }^{1 \dagger}$, Qian Wang ${ }^{1+}$ and Dongsheng $\mathrm{Hu}^{1,4^{*}}$

\begin{abstract}
Background: Associations between transcription factor 7-like 2 (TCF7L2) polymorphisms and type 2 diabetes mellitus (T2DM) have been evaluated extensively in multiple ethnic groups. TCF7L2 has emerged as the strongest T2DM susceptibility gene in Europeans, but the findings have been inconsistent in the Chinese population. The purpose of this meta-analysis was to evaluate the associations between TCF7L2 single nucleotide polymorphisms (SNPs) and T2DM risk in the Chinese population.

Methods: We performed searches in the PubMed, EMBASE, Cochrane, and Chinese databases (CNKI, CQVIP and Wanfang databases) for literature published from January 2007 to February 2012. We reviewed all relevant articles on TCF7L2 polymorphisms and susceptibility to T2DM in the Chinese population written in English and Chinese. Two reviewers extracted data independently using a standardized protocol, and any discrepancies were adjudicated by a third reviewer. Fixed-effects and random-effects meta-analyses were performed to pool the odds ratios (ORs). Publication bias and heterogeneity were examined.

Results: A total of 21 articles were confirmed to be eligible for and included in this meta-analysis: 7 (with 3942 cases and 3502 controls) concerning rs 11196218 (IVS-/+4G>A), 8 (with 3377 cases and 2975 controls) concerning rs290487 (IVS3-/+C>T), and 14 (with 7902 cases and 7436 controls) concerning rs7903146 (IVS3-/+C>T). Overall, the results showed a significant association between rs7903146 and T2DM risk. The pooled ORs were 1.54 for the comparison of T and C alleles (95\% Cl [confidence interval]: 1.37-1.74, $p=1.47 \times 10^{-12}, \mathrm{P}^{2}=25.20 \%$ ) and 1.56 for TC heterozygotes and CC homozygotes (95\% Cl : 1.38-1.76, $\left.p=8.25 \times 10^{-9}, P^{2}=21.00 \%\right)$. The rs 11196218 (IVS4G $>A$ ) and rs290487 (IVS3C>T) SNPs were not associated with T2DM risk.
\end{abstract}

Conclusions: The rs7903146 SNP of the TCF7L2 gene is associated with increased susceptibility to T2DM in the Chinese population as a whole as well as northern Chinese and southern Chinese as subgroups.

Keywords: Type 2 diabetes, T2DM, TCF7L2, SNPs, Meta-analysis

\footnotetext{
* Correspondence: dongsheng_hu@hotmail.com

${ }^{\dagger}$ Equal contributors

'Department of Epidemiology, College of Public Health, Zhengzhou University, Zhengzhou 450000, People's Republic of China

${ }^{4}$ Shenzhen University School of Medicine, Shenzhen 518000, People's

Republic of China

Full list of author information is available at the end of the article
} 


\section{Background}

Type 2 diabetes mellitus (T2DM) is the most common form of diabetes, accounting for $90 \%$ of cases in the world [1]. The incidence and prevalence of T2DM has reached epidemic proportions worldwide. It is estimated that China will contribute almost 38 million patients to the global burden of diabetes by 2025 [2,3]. Moreover, the incidence of T2DM in children has increased dramatically in the past decades [4]. Although T2DM pathogenesis remains to be fully elucidated, genetic susceptibility appears to play a crucial role in the etiology and manifestation of the disease [5-7]. Notably, single nucleotide polymorphisms (SNPs) of the transcription factor 7-like 2 (TCF7L2) gene have been reported to affect T2DM susceptibility by indirectly altering expression of GLP-1 [8], which in addition to insulin, plays a critical role in blood glucose homeostasis [9].

In recent years, numerous studies examining various ethnic groups have demonstrated an association between TCF7L2 genotype and T2DM. However, the results have been inconsistent [10-30]. Five meta-analyses of the association between TCF7L2 SNPs and T2DM have been published [31-35]. The first published in 2007, demonstrated a significant association between the rs7903146 SNP and T2DM in Moroccans (allelic OR $=1.56,95 \%$ CI [confidence interval]: $1.29-1.89, p=2.9 \times 10^{-6}$ ) and Austrians (allelic OR $=1.52$, 95\% CI: $1.29-1.78, p=3.0 \times 10^{-7}$ ) [33]. The second study published in 2009, demonstrated significant associations between four TCF7L2 SNPs (rs7903146, rs12255372, rs7901695 and rs11196205) and T2DM [32]; however, only two articles examining Chinese subjects were included in that meta-analysis. The third meta-analysis, also published in 2009 but which included six studies of Chinese population samples, concluded that there were five TCF7L2 SNPs (rs7903146, rs12255372, rs11196205, rs290487 and rs11196218) associated with T2DM risk among East-Asian people [31]. The fourth study, also published in 2009, focused on differences in the association between rs7903146 and T2DM among different inter-East Asian populations; three of the six studies had examined Chinese population samples [34]. The fifth study was a three-stage analysis designed to identify susceptibility loci for T2DM among East Asians. In the first stage, the researchers performed a meta-analysis of eight T2DM genome-wide association studies (6952 cases and 11,865 controls) for established T2DM risk loci and confirmed a significant association between rs7903146 and T2DM risk among East-Asians (OR $=1.16$, 95\% CI: $1.02-$ 1.31, $p=2.5 \times 10^{-2}$ ) [35]; however, none of those eight studies examined Chinese people.

Sixteen studies $[14,15,18-30]$ examining this question in Chinese population samples have been published since the aforementioned meta-analyses. However, their results have been inconsistent. Thus, the potential association between
TCF7L2 polymorphisms and T2DM in Chinese population remains inconclusive. In this study, we performed an up-to-date meta-analysis to assess the contribution of TCF7L2 polymorphisms to T2DM susceptibility specifically in Chinese people.

\section{Methods}

\section{Primary search strategy}

We conducted a search of the PubMed, EMBASE, Cochrane, and Chinese literature databases (CNKI, CQVIP, and Wanfang databases) for articles published from January 2007 to February 2012. The following subject terms were used: 'TCF7L2', 'genetic polymorphism', 'Chinese', and 'T2D/T2DM'. Only human studies published in English or Chinese were retrieved for consideration. The reference lists of the selected articles were searched to identify any additional relevant articles.

\section{Inclusion criteria and data extraction}

Studies were selected for our analysis if they reported the genotype or allele frequencies of TCF7L2 polymorphisms in Chinese T2D patients and controls. Data were extracted by two reviewers (J. Wang and F. Hu) using a standardized data extraction form. Discrepancies were adjudicated by the third reviewer (L. Li) until a consensus was achieved on each item. A total of 21 eligible articles were included in the meta-analysis.

\section{Statistical analysis}

The Metan module in the STATA 11.0 software package was used to perform the meta-analysis on genotype frequencies. The strength of the association between each SNP and T2DM risk was measured by determining ORs with $95 \%$ CIs. The $\mathrm{Z}$ test was used to determine the statistical significance of pooled ORs. Heterogeneity index $\left(I^{2}, 0-100\right)$ was applied to assess heterogeneity among the studies [36]. In accordance with the Cochrane reviewer's handbook 4.2.2 [37], we accepted a high degree of heterogeneity for study pairs with an $I^{2}>50 \%$. When heterogeneity was not an issue, the fixed effects model was applied with the Mantel-Haenszel method [38]. Otherwise, a random effects model was applied using the DerSimonian and Laird method to calculate the combined OR [39]. Subgroup analyses were performed by region (South or North) for each SNP.

Non-risk and risk alleles were represented by $\mathrm{A} 1$ and A2, respectively. The OR for the risk allele A2, A1/A2 was compared to that for A1/A1, and the OR for A2/A2 was compared to for A1/A1.

The present meta-analysis evaluated the association between three SNPs in TCF7L2 gene and T2D, and the Bonferroni method [40] was employed to adjust for Type I errors. Specifically, the Bonferroni significance threshold was set at $\alpha=0.05 / 3=0.017$ for the 3 SNPs. 
Publication bias was investigated with funnel plots [41]. Furthermore, Egger's regression approach was adopted [42]. Power calculation was performed using PASS ( Power analysis and sample size, by Jerry) software. Population-attributable risk (PAR) was calculated based on the estimated ORs and risk allele frequencies of those SNPs that showed significant association with T2DM risk.

\section{Results}

\section{Inclusion of SNPs for analysis}

Among SNPs previously associated with T2DM risk in the Chinese population, only three were adequately represented in the selected literature and had sufficient low minor allele frequency (MAF) to be included in our metaanalysis: rs7903146, rs11196218, and rs290487. We found that Hapmap data (HapMap Data Rel 27 Phase II+III, Feb09, on NCBI B36 assembly, dbSNP b126) indicate that, in the Chinese population, the linked disequilibrium (LD) structure places D' at 1.0 unit between rs7903146 and rs290487, 1.0 unit between rs7903146 and rs11196218, and 0.059 unit between rs290487 and rs11196218 (Figure 1). Between rs7903146 and rs11196218, $\mathrm{r}^{2}=$ 0.016 , between rs7903146 and rs290487, $\mathrm{r}^{2}=0.013$, and between rs11196218 and rs290487, $r^{2}=0.001$ (see Additional file 1: Table S1). The LD was relatively low among three SNPs in the Chinese population, so we decided to include these SNPs in our meta-analysis.

\section{Characteristics of included studies}

Of 38 studies were identified as the potential candidates, 21 were deemed eligible for the final meta-analysis [10-30]; these 21 articles examined the three included at least one of the SNPs noted above (rs7903146, rs11196218, and rs290487). As reported in detail Additional file 1: Table S2, we examined seven studies (total of 3942 cases and 3502 controls) related to rs11196218 (ISV4-/+G>A), eight studies (total of 3377 cases and 2975 controls) related to rs290487 (ISV3-/+C>T), and fourteen studies (total of 7902 cases and 7436 controls) related to rs7903146 (ISV3 $-/+C>T)$. Our examination of heterogeneity among studies (see Additional file 1: Table S3) revealed a high heterogeneity among studies for rs11196218 and rs290487. As shown in Figure 2 and Additional file 1: Table S4, both Egger's and Begg's tests suggested no publication bias in the associations between rs11196218, rs290487, and rs7903146 and risk of T2DM.

\section{Results of meta-analysis}

The detailed results of our meta-analysis are reported in Additional file 1: Table S3. Notably, we obtained highly significant ORs for rs7903146,

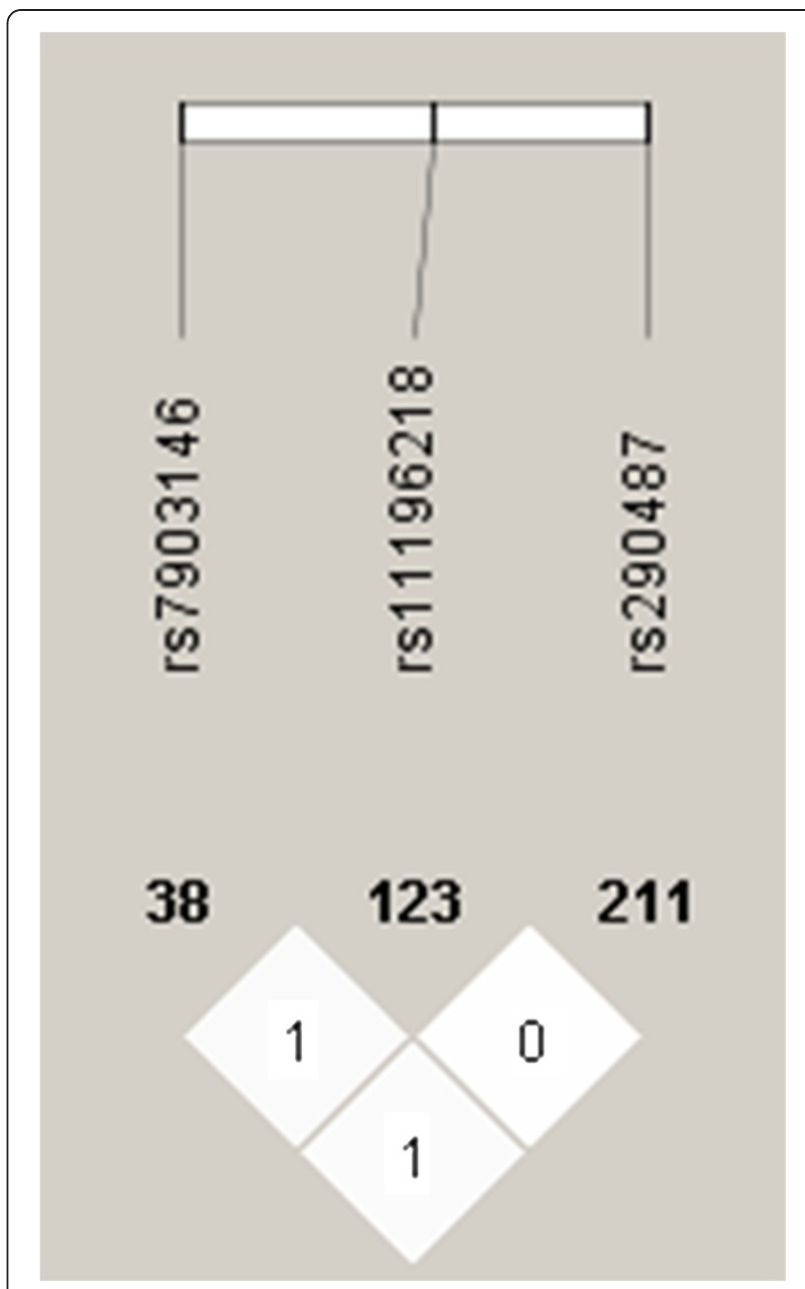

Figure 1 LD plots for rs7903146, rs11196218, and rs290487 in the Chinese population. Based on Hapmap data (HapMap Data Rel 27 Phasell+III, Feb09, on NCBI B36 assembly, dbSNP b126).

including the pooled $\mathrm{OR}$ for the $\mathrm{T}$ allele versus the C allele (OR $=1.54,95 \%$ CI: $1.37-1.74, p=1.47 \times 10^{-12}$, $\left.I^{2}=25.20 \%\right)$ and the OR for heterozygotes versus C homozygotes $(\mathrm{OR}=1.56,95 \% \mathrm{CI}: 1.38-1.76, p=8.25 \times$ $10^{-9}, I^{2}=21.00 \%$ ) (Figure 3 ). The population attributable risk (PAR) was $1.6 \%$ for the risk allele $\mathrm{T}$ and $2.9 \%$ for $\mathrm{CT}$ genotype of rs7903146. We did not find significant associations between the risk alleles for rs11196218 and rs290487 and risk for T2DM.

\section{Results of the subgroup analyses}

When people from the northern and southern regions of China were each considered independently, we found that the allele and genotype of rs7903146 ORs remained significant for both subgroups (see Additional file 1: Table S3). In addition, we found that for rs290487 the pooled OR in subgroup analysis based on the region was 

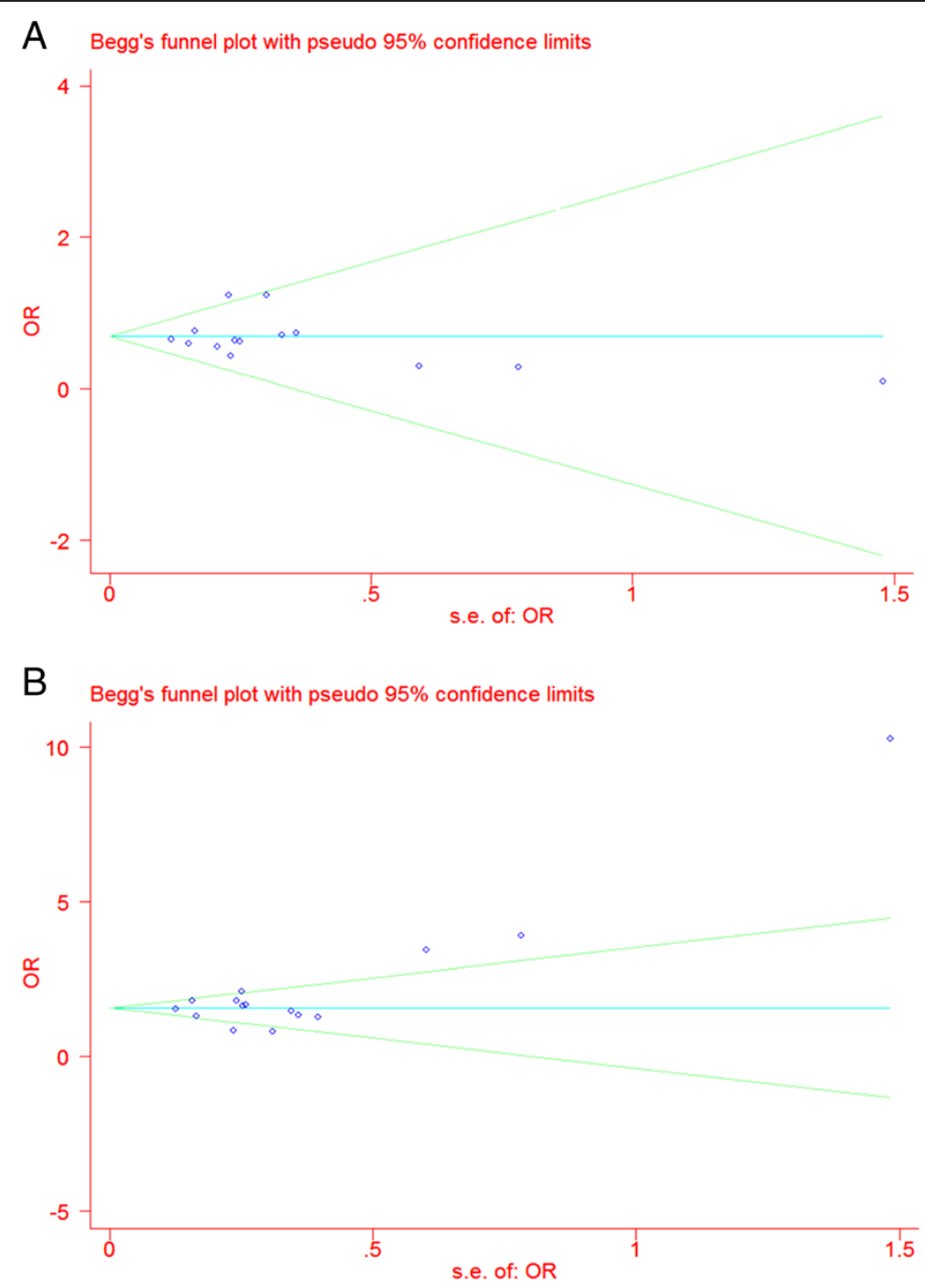

Figure 2 Funnel plot for rs7903146. Panel A: the ORs of allele T versus allele C of rs7903146 overall; Panel B: of the ORs of heterozygotes versus $\mathrm{C}$ homozygotes of rs7903146 overall.

$1.54\left(95 \%\right.$ CI : $\left.1.22-1.94, p=3.25 \times 10^{-5}, I^{2}=0.00 \%\right)$ in the southern region of China.

\section{Sensitivity analysis}

The sensitivity analysis was performed by omitting one study at a time. None of the individual studies influenced the final conclusions for rs290487. Likewise, removal of studies did not affect the conclusion for the risk allele $\mathrm{A}$ and the $\mathrm{A}$ homozygotes versus $\mathrm{G}$ homozygotes comparison for rs11196218, nor the conclusions for the risk allele $\mathrm{T}$ and the heterozygotes versus $\mathrm{C}$ homozygotes comparison for rs7903146. However, when Ying Zhang et al.'s study [24] was omitted, we found that the comparison for heterozygotes versus $\mathrm{G}$ homozygotes of rs11196218, which previously had a $\mathrm{p}$ value of 0.15 , became significant $(\mathrm{OR}=0.87,95 \% \mathrm{CI}: 0.78-0.97, p=$ $\left.0.010, I^{2}=47.80 \%\right)$.

\section{Discussions}

In this study, we evaluated the effects of three wellknown SNPs of the TCF7L2 gene (rs11196218, rs290487, and rs7903146) on the susceptibility for T2DM among Chinese people by reviewing 21 eligible articles. It should be noted that these three SNPs are located in the splicing sites, and thus may affect exons and introns splicing. Our meta-analysis confirmed a significant association between the risk allele in rs7903146 (T) and risk of T2DM in the whole Chinese people regardless of region diversity. When considering the Chinese population as a whole, our data did not support significant 


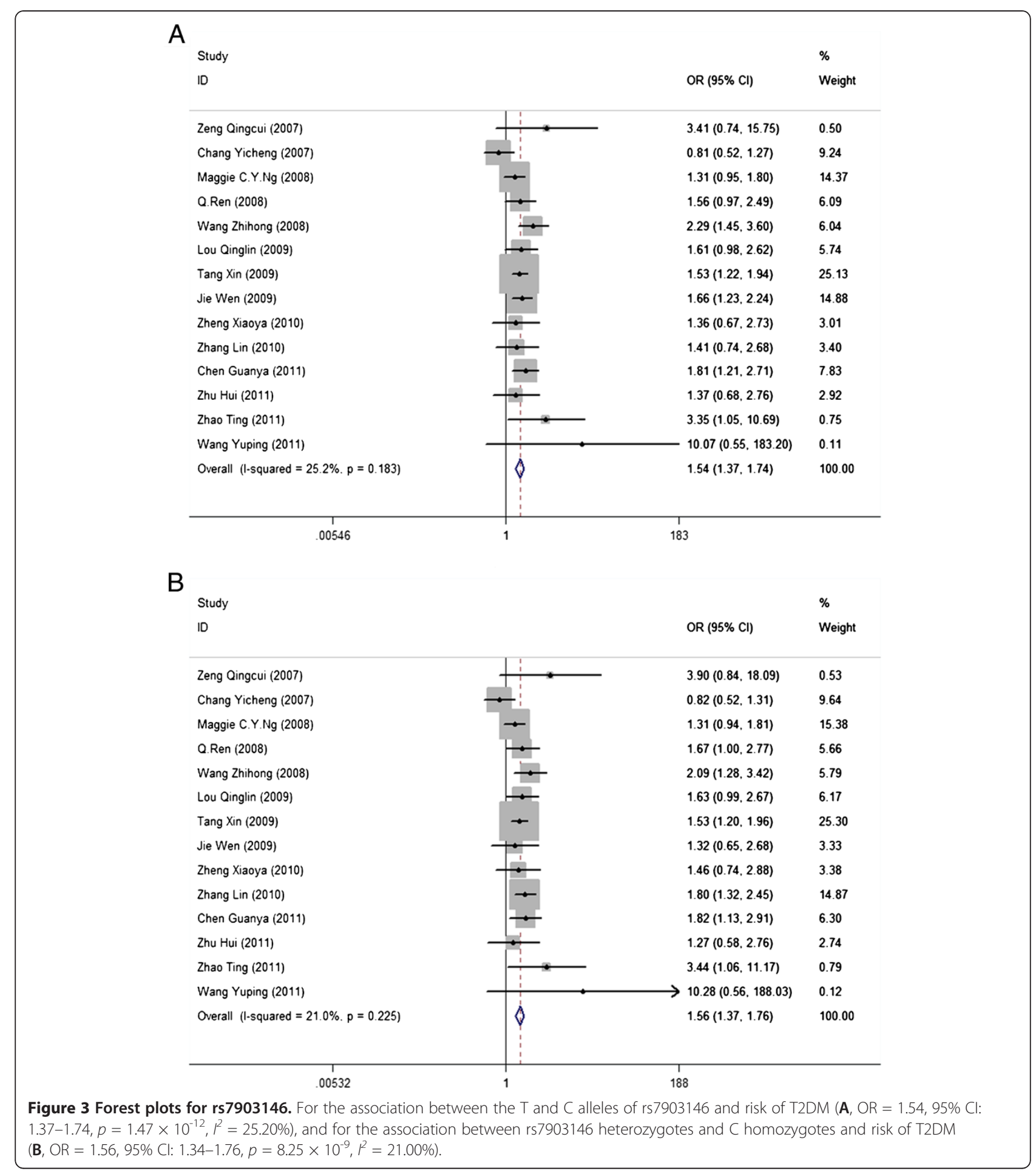

associations for rs290487 with T2DM susceptibility, which contrasts with former results form Luo et al's metaanalysis of studies involving East Asians [31], which showed a combined allelic odds radio for rs290487 (OR = $1.11,95 \%$ CI : 1.03-1.19, $p=0.01$ ). This difference may be due to difference in the populations considered given that the present analysis was focused on studies of Chinese people, while Japanese participants were highly represented in the former study (62.5\% for rs290487). Indeed, as shown in Additional file 1: Table S5 which summarizes the MAFs for Chinese, Japanese, and European populations, MAFs are not uniform across populations (see Additional file 1: Table S5). Specifically, relative to Chinese population, Japanese population have lower MAF for 
rs290487 (0.384 in Japanese compared with 0.439 in mainland China). The present meta-analysis had weak power (only 9.4\%, 6.1\% and 9.9\% power to detect associations of risk allele, CT genotype and CC genotype of rs290487) for detecting associations between rs290487 risk allele or genotype with T2DM risk (see Additional file 1: Table S6).

The significant associations between the rs7903146 SNP in TCF7L2 and T2DM risk found in our study were similar to the findings of a large study (1529 cases and 1439 controls) by Xin Tang et al. in China [25], but contrast with some small sample studies [21,23]. It could be that the small sample studies lacked sufficient statistical power to reveal these associations.

The present results also differ from those Takeuchi et al.'s meta-analysis published in 2009 [34] that focused on the ethnic differences across inter-East Asian populations in that Takeuchi et al.'s combined OR for rs7903146 among Chinese people did not emerge as significant $(\mathrm{OR}=1.21,95 \% \mathrm{CI}: 0.96-1.51, p=0.12)$. This difference is likely due to the fact that in addition to the three Chinese studies included in their meta-analysis, 12 new papers were included in our analysis.

The results in our meta-analysis regarding the rs7903146 risk allele $\mathrm{T}$ and genotype $\mathrm{CT}$ were similar to those reported in 2009 by Tong et al. [32] who examined a variety of ethnic groups including Caucasians, North Europeans, East Asians, Indians, Africans, and others. However, our genotype TT finding differed from Tong et al.'s results. Given the ethnically broad inclusion of Tong et al's study and the ethnically narrow inclusion of ours, there are several population-related reasons for this difference. First, the risk allele frequency in the Chinese population is much lower than that in Europeans (the minor allele frequencies was 0.024 and 0.035 in the Chinese and Japanese populations, respectively; it was 0.279 in European populations, listed in Additional file 1: Table S5. Second, there was also a substantial difference in LD structure between Chinese and European population (see Additional file 1: Table S1 for $\mathrm{r}^{2}$ values based on HapMap data). These differences in genetic structure between the Chinese and European populations may explain why this SNP produced different results between studies involving different ethnic groups. Due to the lower MAF of rs7903146 in Chinese populations, the estimated PARs for the $\mathrm{T}$ allele $(1.7 \%)$ and the $\mathrm{CT}$ genotype (3.2\%) were much lower in our analysis than the PARs reported for populations of European ancestry (17-28\%) [43].

When we separated northern Chinese from southern Chinese, the pattern of our risk results changed for rs290487. Specifically, when southern Chinese subjects were analyzed separatedly, we obtained significant OR for the $\mathrm{C}$ homozygotes versus $\mathrm{T}$ homozygotes comparison for rs290487, pointing to an increased T2DM risk for C homozygotes.

Heterogeneity is a potential problem when interpreting the results of meta-analyses [44]. In our meta-analysis, high heterogeneity persisted in studies examining associations between the rs290487 the rs11196218 SNPs and T2DM risk. Because our subject pool was limited entirely to mainland Chinese persons, the ethnic heterogeneity of our study was limited. In our subgroup analysis, heterogeneity was not reduced for either of the regional subgroups. Nevertheless, gender and age likely contributed to the heterogeneity of our study population.

Violations of the Hardy-Weinberg assumptions can cause deviations from expectation due to improper sampling. The genotypes of both cases and controls did not deviate significantly from the Hardy-Weinberg equilibrium in any of the 21 reported deemed eligible for inclusion in our study.

Pooling risk estimates from multiple studies can increase statistical power. To our knowledge, our metaanalysis is the largest such study of this question selectively involving Chinese subjects. However, there are several limitations inherent in performing a meta-analysis of this kind that shoud be noted. First, due to the lower MAF of some TCF7L2 SNPs in the Chinese population, the literature examing the relationship between these SNPs and T2DM in Chinese subjects specifically is relatively limited. Second, the sample sizes of some of the included studies were relatively small. Third, since our analysis involves previously published source data, the extent of our evalystion of potential interactions of these SNPs with T2DM is limited. Finally, given the lack of full information about gender and age of the subjects across the studies, it was not possible to perform a stratified analysis or interaction analysis based on age or gender.

\section{Conclusions}

The rs7903146 SNP of the TCF7L2 gene is associated with increased susceptibility to T2DM in the Chinese population as a whole as well as northern Chinese and southern Chinese as subgroups.

\section{Additional file}

Additional file 1: Table S1. LD (as r 2 ) between TCF7L2 SNPs in this meta-analysis in Chinese v.s. Caucasians. Table S2. Main characteristics of the 21 studies deemed eligible for meta-analysis. Table S3. Results of meta-analysis for TCF7L2 polymorphisms and the T2DM susceptibility. Table S4. Results of Egger's test. Table S5. MAFs for the three presently examined SNPs analyzed in the Chinese, Japanese, and European populations. Table S6. Power calculation for the present meta-analysis.

\section{Abbreviations}

SNP: Single nucleotide polymorphism; TCF7L2: Transcription factor 7-like 2; Cl: Confidence interval; OR: Odds ratio; BMI: Body mass index; T2DM: Type 2 
diabetes; MAF: Minor allele frequency; PAR: Population attributable risk; LD: Linkage disequilibrium.

\section{Competing interests}

The authors have no competing interests to declare.

\section{Authors' contributions}

$\mathrm{DH}$ produced the design of the study, analyzed data, and drafted the manuscript. JW contributed to the design of the study, analyzed data, and helped write the manuscript. JW, TF, JZ, LY, YW and QW conducted database searches. JW, FH, and LL conducted data extraction and statistical analyses. All authors approved the final manuscript.

\section{Acknowledgements}

This work was supported by grants from China's National Natural Science Foundation (contact NO. 81072359). We sincerely thank Dr. Jing (Sophia) Xie (Health Services and Ocular Epidemiology, Research Unit Centre for Eye Research Australia, Department of Ophthalmology, The University of Melbourne), Marios Constantinou (Project Manager and Clinical Trials Coordinator, Health Services and Ocular Epidemiology, Research Unit), and Bonnie Adele Sturrock (Health Services and Ocular Epidemiology Research) for providing critical feedback on this manuscript. We sincerely thank Dr. Chen Mao (Division of Epidemiology, School of Public Health and Primary Care, The Chinese University of Hong Kong), Dr Zuyao (Joey) Yang (Division of Epidemiology, School of Public Health and Primary Care, The Chinese University of Hong Kong) for their enomous help.

\section{Author details}

'Department of Epidemiology, College of Public Health, Zhengzhou University, Zhengzhou 450000, People's Republic of China. ${ }^{2}$ Department of Epidemiology, Public Health College, Harbin Medical University, Harbin 150086, People's Republic of China. ${ }^{3}$ Military Hospital of Henan Province, Zhengzhou 450000, People's Republic of China. ${ }^{4}$ Shenzhen University School of Medicine, Shenzhen 518000, People's Republic of China.

Received: 9 April 2012 Accepted: 7 January 2013

Published: 12 January 2013

\section{References}

1. Stumvoll M, Goldstein BJ, van Haeften TW: Type 2 diabetes: principles of pathogenesis and therapy. Lancet 2005, 365(9467):1333-1346.

2. Roglic G, King H: Diabetes mellitus in Asia. Hong Kong Med J 2000, 6(1):10-11.

3. King H, Aubert RE, Herman WH: Global burden of diabetes, 1995-2025: prevalence, numerical estimates, and projections. Diabetes Care 1998, 21(9):1414-1431.

4. American Diabetes Association: Type 2 diabetes in children and adolescents. Diabetes Care 2000, 23(3):381-389.

5. Flores-Martinez SE, Islas-Andrade S, Machorro-Lazo MV, Revilla MC, Juarez RE, Mujica-Lopez KI, Moran-Moguel MC, Lopez-Cardona MG, SanchezCorona J: DNA polymorphism analysis of candidate genes for type 2 diabetes mellitus in a Mexican ethnic group. Ann Genet 2004, 47(4):339-348

6. Radha V, Mohan V: Genetic predisposition to type 2 diabetes among Asian Indians. Indian J Med Res 2007, 125(3):259-274.

7. Elbein SC: The genetics of human noninsulin-dependent (type 2) diabetes mellitus. J Nutr 1997, 127(9):1891S-1896S.

8. Holst JJ: The physiology of glucagon-like peptide 1. Physiol Rev 2007, 87(4):1409-1439.

9. Yi F, Brubaker PL, Jin T: TCF-4 mediates cell type-specific regulation of proglucagon gene expression by beta-catenin and glycogen synthase kinase-3beta. J Biol Chem 2005, 280(2):1457-1464.

10. Ren $Q$, Han XY, Wang F, Zhang XY, Han LC, Luo YY, Zhou XH, Ji LN: Exon sequencing and association analysis of polymorphisms in TCF7L2 with type 2 diabetes in a Chinese population. Diabetologia 2008, 51(7):1146-1152.

11. Chang YC, Chang TJ, Jiang YD, Kuo SS, Lee KC, Chiu KC, Chuang LM: Association study of the genetic polymorphisms of the transcription factor 7-like 2 (TCF7L2) gene and type 2 diabetes in the Chinese population. Diabetes 2007, 56(10):2631-2637.
12. Ng MC, Tam CH, Lam VK, So WY, Ma RC, Chan JC: Replication and identification of novel variants at TCF7L2 associated with type 2 diabetes in Hong Kong Chinese. J Clin Endocrinol Metab 2007, 92(9):3733-3737.

13. Ng MC, Park KS, Oh B, Tam CH, Cho YM, Shin HD, Lam VK, Ma RC, So WY, Cho YS, et al: Implication of genetic variants near TCF7L2, SLC30A8, HHEX, CDKAL1, CDKN2A/B, IGF2BP2, and FTO in type 2 diabetes and obesity in 6,719 Asians. Diabetes 2008, 57(8):2226-2233.

14. Zheng X, Ren W, Zhang S, Liu J, Li S, Li J, Yang P, He J, Su S, Li P: Association of type 2 diabetes susceptibility genes (TCF7L2, SLC30A8, PCSK1 and PCSK2) and proinsulin conversion in a Chinese population. Mol Biol Rep 2012, 39(1):17-23.

15. Yu M, Xu XJ, Yin JY, Wu J, Chen X, Gong ZC, Ren HY, Huang Q, Sheng FF, Zhou HH, et al: KCNJ11 Lys23Glu and TCF7L2 rs290487(C/T) polymorphisms affect therapeutic efficacy of repaglinide in Chinese patients with type 2 diabetes. Clin Pharmacol Ther 2010, 87(3):330-335.

16. Lou QL: The association study between SNPs in TCF7L2 and the susceptibility of T2D in Chinese Han population. Chin J Diabetes 2007, 17(12):895-898.

17. Zeng QC: Factor 7-like 2 (TCF7L2) Gene in Type2 Diabetes Mellitus of Chinese in Chengdu Area. In Master's thesis: University of Sichuan, Clinical Medicine Department; 2007.

18. Chen GY: Relationship between TCF7L2 polymorphism with T2D and related factors. Journal of Xianning University (Medical Sciences) 2011, 25(2):103-106.

19. Zou YL: Relationship between rs290487-T/C polymorphism of TCF7L2 gene and T2D in Kunming Han population. China Modern Doctor 2009, 47(29):11-12.

20. Wang ZH: Association of polymorphism in TCF7L2 and SLC30A8 with T2D in Chinese Han population. In Phd thesis: Chongqing University, Clinical Medicine Department; 2008.

21. Zhang L: Replication study for the association of TCF7L2 rs 12255372 and rs7903146 with susceptibility to T2D in a Chinese Han population. In Master's thesis: University of South China, Clinical Medicine Department; 2010.

22. Ma C: Relationship of rs13266634 and rs11196218 polymorphism in SLC30A8 (solute carrier family 30, member 8) and TCF7L2 (transcription factor 7 like 2) gens with T2D in Southern Chinese Han population. In Master's thesis: Jiangsu University, Clinical Medicine Department; 2006.

23. Zhang Y: Study on the relationship between the rs 290487 polymorphism of TCF7L2 gene and T2D mellitus. In Mater's thesis: Shandong University, Clinical Medicine Department; 2009.

24. Zhang Y: Association of polymorphism in TCF7L2 with T2D in Chinese Han population. In Master's thesis: Zhongnan University, Clinical Medicine Department; 2008.

25. Xin T: The study of single nucleotide polymorphism of susceptibility genes of type two diabetes in Chinese Han population. In Master's thesis: Chongqing Medical University, Clinical Laboratory Department; 2009.

26. Zhu H: Common risks of the TCF7L2 gene with T2D and impaired glucose regulation in a Chinese Han population. In Master's thesis: Anhui Medical University, Clinical Medicine Department; 2011.

27. Qiao H, Zhang X, Zhao X, Zhao Y, Xu L, Sun H, Fu S: Genetic variants of TCF7L2 are associated with type 2 diabetes in a northeastern Chinese population. Gene 2012, 495(2):115-119.

28. Zhao T: The association of transcription factor 7 like 2 polymorphisms with the early phase function of $\beta$-cell in T2D mellitus. Med J Qilu 2011, 26(5):417-20.

29. Wen J, Ronn T, Olsson A, Yang Z, Lu B, Du Y, Groop L, Ling C, Hu R: Investigation of type 2 diabetes risk alleles support CDKN2A/B, CDKAL1, and TCF7L2 as susceptibility genes in a Han Chinese cohort. PLOS One 2010, 5(2):e9153.

30. Wang $Y$, Zhu H, Jin $X$, LV Y, Cui Z, Jin $Y$, Zhang Z, Yang K: The association study between T2D and rs7903146 polymorphisms in TCF7L2 in Korean and Han Chinese population living in Yanbian [abstract]. China Genetics Conference Abstracts 2011, 8:s212.

31. Luo Y, Wang H, Han X, Ren Q, Wang F, Zhang X, Sun X, Zhou X, Ji L: Metaanalysis of the association between SNPs in TCF7L2 and type 2 diabetes in East Asian population. Diabetes Res Clin Pract 2009, 85(2):139-146.

32. Tong $Y$, Lin $Y$, Zhang $Y$, Yang J, Zhang $Y$, Liu H, Zhang B: Association between TCF7L2 gene polymorphisms and susceptibility to type 2 diabetes mellitus: a large Human Genome Epidemiology (HuGE) review and meta-analysis. BMC Med Genet 2009, 10:15. 
33. Cauchi S, El AY, Choquet H, Dina C, Krempler F, Weitgasser R, Nejjari C, Patsch W, Chikri M, Meyre D, et al: TCF7L2 is reproducibly associated with type 2 diabetes in various ethnic groups: a global meta-analysis. $J \mathrm{Mol}$ Med (Berl) 2007, 85(7):777-782.

34. Takeuchi M, Okamoto K, Takagi T, Ishii H: Ethnic difference in patients with type 2 diabetes mellitus in inter-East Asian populations: a systematic review and meta-analysis focusing on gene polymorphism. J Diabetes 2009, 1(4):255-262.

35. Cho YS, Chen CH, Hu C, Long J, Ong RT, Sim X, Takeuchi F, Wu Y, Go MJ, Yamauchi T, et al: Meta-analysis of genome-wide association studies identifies eight new loci for type 2 diabetes in east Asians. Nat Genet 2012, 44(1):67-72.

36. Higgins JP, Thompson SG, Deeks JJ, Altman DG: Measuring inconsistency in meta-analyses. BMJ 2003, 327(7414):557-560.

37. Ling W, Farrell M, Ali R: Cochrane systematic reviews: time for an introduction and appraisal. Drug Alcohol Depend 2004, 73(3):217-218.

38. Mantel N, HAENSZEL W: Statistical aspects of the analysis of data from retrospective studies of disease. J Natl Cancer Inst 1959, 22(4):719-748.

39. Midgette AS, Wong JB, Beshansky JR, Porath A, Fleming C, Pauker SG: Cost-effectiveness of streptokinase for acute myocardial infarction: a combined meta-analysis and decision analysis of the effects of infarct location and of likelihood of infarction. Med Decis Making 1994, 14(2):108-117.

40. Noble WS: How does multiple testing correction work? Nat Biotechnol 2009, 27(12):1135-1137.

41. Light RJ: Summing up: the science of reviewing research. Cambridy, MA: Harvard University Press; 1984.

42. Egger M, Davey SG, Schneider M, Minder C: Bias in meta-analysis detected by a simple, graphical test. BMJ 1997, 315(7109):629-634.

43. Helgason A, Palsson S, Thorleifsson G, Grant SF, Emilsson V, Gunnarsdottir S, Adeyemo A, Chen Y, Chen G, Reynisdottir I, et al: Refining the impact of TCF7L2 gene variants on type 2 diabetes and adaptive evolution. Nat Genet 2007, 39(2):218-225.

44. Nakaoka H, Inoue I: Meta-analysis of genetic association studies: methodologies, between-study heterogeneity and winner's curse. J Hum Genet 2009, 54(11):615-623.

doi:10.1186/1471-2350-14-8

Cite this article as: Wang et al:: Meta-analysis of associations between TCF7L2 polymorphisms and risk of type 2 diabetes mellitus in the Chinese population. BMC Medical Genetics 2013 14:8

\section{Submit your next manuscript to BioMed Central and take full advantage of:}

- Convenient online submission

- Thorough peer review

- No space constraints or color figure charges

- Immediate publication on acceptance

- Inclusion in PubMed, CAS, Scopus and Google Scholar

- Research which is freely available for redistribution 